\title{
Polycystic ovary syndrome and the risk of obstructive sleep apnea: a meta-analysis and review of the literature
}

\author{
Nafiye Helvaci ${ }^{1}$, Erdem Karabulut ${ }^{2}$, Ahmet Ugur Demir ${ }^{3}$ and Bulent Okan Yildiz \\ 'Department of Internal Medicine, Division of Endocrinology and Metabolism, Hacettepe University \\ School of Medicine, Ankara, Turkey \\ 2Department of Biostatistics, Hacettepe University School of Medicine, Ankara, Turkey \\ ${ }^{3}$ Department of Chest Diseases, Hacettepe University School of Medicine, Ankara, Turkey
}

Correspondence

should be addressed

to $B$ O Yildiz

Email

yildizbo@yahoo.com

\begin{abstract}
Background and Objective: Polycystic ovary syndrome (PCOS) has been reported to be associated with the development of obstructive sleep apnea (OSA). The objective of this meta-analysis is to assess the relationship between PCOS and OSA.

Methods: A literature search was conducted to identify studies linking PCOS with the risk of OSA. Studies in which the presence of OSA was confirmed with overnight polysomnography were included. Random effects models were used to calculate pooled relative risks.

Results: Eight studies conducted in adults and five studies conducted in adolescents were identified. The pooled OSA prevalence was 0.22 ( $95 \%$ confidence interval $(\mathrm{Cl}): 0.08-0.40)$ in PCOS patients. The pooled prevalence of OSA was higher in adults $(0.32,95 \% \mathrm{Cl}$ : $0.13-0.55)$ than adolescents $(0.08,95 \% \mathrm{Cl}: 0.00-0.30)$. Risk of OSA was significantly increased in adult patients with PCOS (odds ratio (OR) 9.74, 95\% Cl: 2.76-34.41). Risk of OSA was not significantly increased in adolescents (OR: 4.54, 95\% Cl:0.56-36.43). Conclusions: These findings demonstrate a significant association between PCOS and OSA in adult patients. Considering the increased risk for long-term cardiometabolic disorders associated with both PCOS and OSA, it is important to diagnose and treat OSA in patients with PCOS.
\end{abstract}

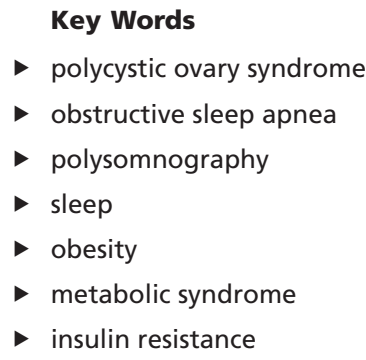

Endocrine Connections (2017) 6, 437-445

\section{Introduction}

Polycystic ovary syndrome (PCOS) is among the most common endocrine disorders in women of reproductive age. The prevalence of PCOS ranges from 5 to $15 \%$ depending on the diagnostic criteria used $(1,2)$. There is a general agreement that the diagnosis of PCOS requires the presence of at least two of the following three abnormalities: chronic anovulation, elevated levels of serum androgens or clinical manifestations of hyperandrogenism and polycystic ovaries on ultrasonography (3). PCOS has its onset in early reproductive years and typically presents with irregular menstrual cycles, infertility and clinical signs of hyperandrogenism including hirsutism, acne and androgenic alopecia. Beyond initial clinical presentation, it is also associated with a substantial increase in risk for long-term cardiometabolic disorders including obesity, impaired glucose tolerance, type 2 diabetes, dyslipidemia, hypertension, coronary and other vascular disorders (4). There is also a growing body of literature suggesting that obstructive sleep apnea (OSA) can be added to the list of cardiometabolic disorders in PCOS.

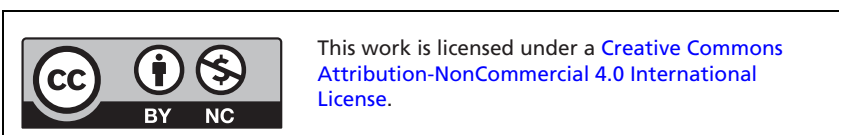


OSA is a relatively common and chronic sleep disorder characterized by recurrent complete (apnea) or partial (hypopnea) upper airway obstructions during sleep leading to intermittent hypoxia, cortical microarousals, sleep fragmentation and increased sympathetic neural activity (5). The overnight polysomnography is the standard diagnostic test for OSA. Obstructive sleep apnea severity is typically assessed with apnea-hypopnea index (AHI), which is the total number of apneas and hypopneas per hour of sleep, recorded during polysomnography. The health consequences of OSA include excessive daytime sleepiness, cognitive dysfunction, decreased quality of life and increased risk of accidents. There is also strong evidence suggesting that OSA contributes to the development of hypertension, cardiovascular disease and abnormalities in glucose metabolism $(6,7$, $8)$. According to the available population-based studies, OSA associated with accompanying daytime sleepiness occurs in approximately $3-7 \%$ of adult men and $2-5 \%$ of adult women in general population (9). Obesity, increased age and male gender are among the most important risk factors implicated for OSA (9).

In a number of studies, prevalence of OSA in women with PCOS has been reported to be considerably higher than that in general population $(10,11,12)$. Although the pathophysiological mechanisms are not fully understood, obesity, insulin resistance and hyperandrogenemia, which are the main features of PCOS, are all proposed to play a role in the development of OSA in patients with PCOS.

The objective of this meta-analysis was to examine if there is an association between PCOS and the occurence of OSA and to assess the potential influence of obesity, insulin resistance and androgen excess on this association.

\section{Materials and methods}

\section{Data sources and study selection}

A systematic literature search was performed independently by two investigators to identify all studies published before September 2015 that investigated the association between PCOS and OSA. Electronic databases, including PubMed and Web of Science were searched using a combination of following terms: obstructive sleep apnea or OSA or sleep disordered breathing or SDB or sleep and polycystic ovary syndrome or polycystic ovarian syndrome or PCOS. The reference lists of relevant publications were also checked for additional publications that might be appropriate to include in the analysis.

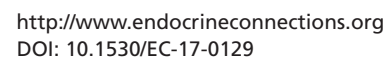

\section{Inclusion and exclusion criteria}

Original studies reported in English and published as full text articles were included in the analysis. There were no country restrictions. All included studies used an objective measurement to assess the presence of OSA. Those studies using questionnaire-based methods for the presence of OSA were excluded.

\section{Data extraction}

Information was collected for each publication concerning the publication year, first author's name, study design, number, gender and age of the participants, diagnostic criteria for PCOS, method that is used to assess OSA, diagnostic criteria for OSA, mean levels of AHI, body mass index (BMI), waist-to-hip ratio, and levels of total and free testosterone, fasting glucose and fasting insulin.

\section{Statistical analysis}

Meta-analysis was performed using metafor package, version 1.9-5 in R 3.1.2 software. Freeman-Tukey double arcsine transformation that provides the confidence limits of proportions between 0 and 1 was applied for normalizing and variance stabilizing of the proportions sampling distribution. Odds ratio was used to assess the relationship between OSA and PCOS. Cochrane $Q$ test and Higgins $I^{2}$ test were used to assess the heterogeneity among studies. When significant heterogeneity was observed among studies, random effects analysis based on the DerSimonian and Laird method was used to estimate the pooled odds ratios and 95\% confidence intervals. Sensitivity analysis was performed by removing one study at a time and the meta-analysis was repeated to assess whether any individual study significantly affected pooled estimates.

\section{Results}

A flow chart indicating the procedure for identifying the studies is presented in Fig. 1. Eight studies conducted in adults and five studies conducted in adolescents including a total of 404 PCOS patients and 611 control subjects were identified. Table 1 summarizes the characteristics of the studies included in the meta-analysis.

One study did not specifiy the criteria used to diagnose PCOS (12). In two studies, PCOS was defined

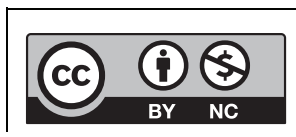

This work is licensed under a Creative Commons Attribution-NonCommercial 4.0 International License. 


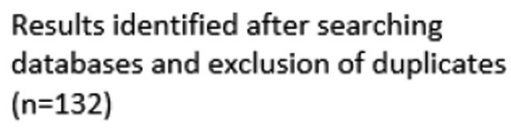

\section{Studies excluded after reading}

titles and abstracts

Irrelevant topic $(\mathrm{n}=92)$

Review, editorial $(n=21)$

\section{Studies excluded}

PSG was not used to

assess the presence of OSA $(n=4)$

Investigated the impact of

treatment, but not the

prevalence of OSA in patients

with PCOS ( $n=1)$

\section{Full articles identified and reviewed} $(n=14)$

Included in meta-analysis ( $n=13$ )

Studies excluded Included subjects who had been part of the previous study group of the investigator $(n=1)$

\section{Figure 1}

Flow chart showing the procedure for identifying the studies included in the meta-analysis. by the presence of any two of the following three features: chronic oligomenorrhea, biochemical or clinical evidence of hyperandrogenism and polycystic ovaries on ultrasonography $(13,14)$. In the rest of the included studies, presence of both chronic anovulation/oligoamenorrhea and hyperandrogenemia was the main criteria for PCOS diagnosis.

OSA was assessed by standard overnight polysomnography in all included studies. OSA was defined according to respiratory distress index, the number of apneas, hypopneas and respiratory effort-related arousals (RERA) per hour of sleep, in two studies $(12,13)$. In the rest of the included studies, AHI was used to define OSA.

The pooled OSA prevalence was 0.22 (95\% CI: 0.08-0.40) in PCOS patients as presented in Fig. 2. The pooled prevalence of OSA was higher in adults $(0.32$, 95\% CI: 0.13-0.55) than that in adolescents (0.08, 95\% CI: 0.00-0.30). Data needed to calculate odds ratio were presented in only six studies (four studies in adults, two studies in adolescents). According to these studies, pooled risk of OSA is 8.3 (95\% CI: 3.30-20.87) times higher in PCOS patients (Fig. 3). The pooled risk of OSA was significantly increased in adults (OR: 9.74, 95\% CI: 2.76-34.41), but because of the wide confidence interval, the results should be interpreted with caution.
Risk of OSA was not significantly increased in adolescents (OR: 4.54, 95\% CI: 0.56-36.43).

We observed significant heterogeneity among studies. $I^{2}$ value was $94.1 \%(Q=201.7, P<0.001)$ for all included studies. The magnitude of the heterogeneity remained high when the analysis restricted to studies enrolling only adults or only adolescents. $I^{2}$ values were $94.2 \%(Q=120.9$, $P<0.001)$ and $92.9 \%(Q=56.5, P<0.001)$ for adult and adolescent studies, respectively. Sensitivity analysis showed that no study had a substantial effect on betweenstudy heterogeneity in adults. In adolescent studies, when the study by Nandalike and coworkers (15) was removed from the analysis, between-study heterogeneity significantly decreased $\left(I^{2}=0 \%, Q=0.29, P=0.961\right)$.

At least 10 studies should be available to evaluate the influence of study-related factors on outcome and the potential source of heterogeneity by meta-regression analysis (16). In this meta-analysis, because of the very small number of the articles in which the descriptive statistics of the potential confounders (BMI, waist circumference, waist-to-hip ratio, total/free testosterone, fasting glucose, fasting insulin) were presented, metaregression analysis could not be performed to assess the impact of these variables on the association between PCOS and OSAS. http://www.endocrineconnections.org DOI: 10.1530/EC-17-0129
() 2017 The authors Published by Bioscientifica Ltd
This work is licensed under a Creative Commons Attribution-NonCommercial 4.0 International License. 
Table 1 Characteristics of the studies on polycystic ovary syndrome (PCOS) and the risk of obstructive sleep apnea (OSA), which were included in the meta-analysis.

\begin{tabular}{|c|c|c|c|c|}
\hline First author, year & Country & Diagnostic criteria for PCOS & $\begin{array}{l}\text { Assessment } \\
\text { of OSA }\end{array}$ & $\begin{array}{l}\text { Diagnostic criteria for } \\
\text { OSA }\end{array}$ \\
\hline Vgontzas, 2001 & USA & Chronic anovulation + hyperandrogenemia + PCO & NP & $\begin{array}{l}\text { AHI } \geq 10 \text { along with } \\
\text { symptoms }\end{array}$ \\
\hline Fogel, 2001 & USA & Chronic oligomenorrhea + hyperandrogenemia & NP & $\begin{array}{l}\text { AHI }>5 \text { along with } \\
\text { symptoms }\end{array}$ \\
\hline Vgontzas, 2006 & USA & Chronic anovulation + hyperandrogenemia + PCO & NP & Not specified \\
\hline Tasali, 2008 & USA & Oligo/amenorrhea + hyperandrogenemia + hyperandrogenism & NP & $\mathrm{AHI} \geq 5$ \\
\hline Gopal, 2002 & USA & Not specified & NP & $\begin{array}{l}\text { RDI } \geq 5 \text { along with } \\
\text { symptoms }\end{array}$ \\
\hline Chatterjee, 2014 & India & $\begin{array}{l}\text { Any } 2 \text { of the following three: chronic oligomenorrhea, } \\
\text { biochemical or clinical hyperandrogenism, PCO }\end{array}$ & NP & $\begin{array}{l}\text { RDI } \geq 5 \text { along with } \\
\text { symptoms or } \mathrm{RDI}>15\end{array}$ \\
\hline Tock, 2014 & Brazil & $\begin{array}{l}\text { Any } 2 \text { of the following three: chronic oligomenorrhea, } \\
\text { biochemical or clinical hyperandrogenism, PCO }\end{array}$ & NP & $\mathrm{AHI} \geq 5$ \\
\hline Yang, 2009 & Taiwan & Oligomenorrhea + hyperandrogenemia + PCO & NP & $\mathrm{AHI} \geq 5$ \\
\hline De Sousa, 2010 & Germany & $\begin{array}{l}\text { Chronic anovulation + clinical or biochemical } \\
\text { hyperandrogenism }\end{array}$ & NP & Not specified \\
\hline De Sousa, 2011 & Germany & $\begin{array}{l}\text { Chronic anovulation + clinical or biochemical } \\
\text { hyperandrogenism }\end{array}$ & NP & Not specified \\
\hline De Sousa, 2012 & Germany & $\begin{array}{l}\text { Chronic anovulation + clinical or biochemical } \\
\text { hyperandrogenism }\end{array}$ & NP & Not specified \\
\hline De Sousa, 2012 & Germany & $\begin{array}{l}\text { Chronic anovulation + clinical or biochemical } \\
\text { hyperandrogenism }\end{array}$ & NP & Not specified \\
\hline Nandalike, 2012 & USA & Oligo/amenorrhea + clinical or biochemical hyperandrogenism & NP & $\mathrm{AHI}>5$ \\
\hline
\end{tabular}

\section{Discussion}

Over the past few years, the role of PCOS in the development of OSA has been increasingly studied. Unfortunately, the current evidence is inconclusive because almost all studies on this topic have a crosssectional design with a small sample size, making it difficult to establish a causal link between PCOS and OSA. In order to determine the possible association between these two disorders and to provide an objective basis for clinical recommendations, we conducted a meta-analysis.

In this meta-analysis, we observed that risk of OSA is not significantly increased in adolescent patients with PCOS. In studies conducted by De Sousa and coworkers, AHI levels of adolescents with PCOS were not high enough to meet the OSA criteria $(17,18,19,20)$. Although respiratory polysomnographic variables were not different between obese adolescents with PCOS and normal-weight or obese controls, they observed some differences in sleep architecture. Obese girls with PCOS had significantly lower sleep efficiency and higher sleep onset latency than both obese and normal-weight controls $(17,20)$. In contrast, Nandalike and coworkers reported increased prevalence of OSA in adolescent girls with PCOS compared to age- and BMI-matched controls
(16/28 (57\%) vs 4/28 (14.3\%), $P<0.01)(15)$. This was a retrospective chart review study and study population included adolescent girls with PCOS who were referred for polysomnography because of sleep-related complaints. Besides that, the participants in the study of Nandalike were more obese than the ones in the studies of de Sousa. There were also racial and ethnic differences between the study groups (predominantly African American/Hispanic, African American, and Hispanic ethnicities in the study of Nandalike compared to a majority of Whites in the studies of de Sousa). Thus, the differences in the study populations, may be the cause of the discrepancy in the results of these studies. In another study, Nandalike and coworkers reported higher prevalence of sleep-disordered breathing and excessive daytime sleepiness in adolescent girls with PCOS compared with age-, sex-, race- and BMI $Z$-score-matched controls, but this study was based on screening questionnaires (21).

In adult patients with PCOS, we observed that there is 9.74 times higher risk for OSA. This is consistent with the results of studies in which questionnaires are used to assess sleep disturbances in adult patients with PCOS. Tasali and coworkers assessed sleep quality, risk of sleep apnea and daytime sleepiness using three questionnaires and found that among 40 women with PCOS, 75\% were at high risk

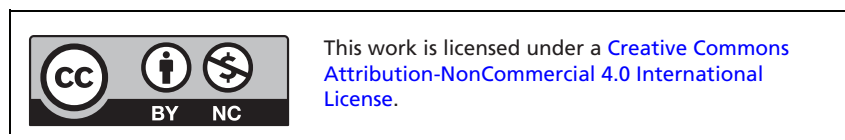




\section{Age PCOS/control \\ $30.4 \pm 0.9 / 32.1 \pm 0.3$ \\ $31.1 \pm 1.3 / 32.3 \pm 1.3$ \\ $29.6 \pm 0.9 / 35.7 \pm 1.0$ * \\ $29.7 \pm 0.7 / 30.7 \pm 1.1$ \\ Premenopausal/no control}

Adult/no control

$28.3 \pm 6.8 /$ no control

$29.1 \pm 1.4 / 31.6 \pm 3.9$

$15.2 \pm 1.3 / 15.0 \pm 1.0$

$15.7 \pm 1.9 / 15.3 \pm 1.0$

$17.8 \pm 1.1 / 19.4 \pm 0.7$ *

$\S / 15.23 \pm 1.09$

$16.8 \pm 1.9 / 17.1 \pm 1.8$

\begin{tabular}{c}
\hline BMI PCOS/control \\
\hline $38.7 \pm 1.1 / 26.4 \pm 0.3^{*}$ \\
$36.9 \pm 1.3 / 36.9 \pm 1.4$ \\
$38.7 \pm 1.4 / 36.9 \pm 1.0$ \\
$39.2 \pm 1.0 / 36.0 \pm 1.5$ \\
$31.4-67$
\end{tabular}

\#\#

$32.9 \pm 7.7$

$21.7 \pm 0.57 / 20.9 \pm 0.58$

$31.7 \pm 6.2 / 34.8 \pm 8.7$

$36.2 \pm 6.2 / 34.4 \pm 6.5$

$32.7 \pm 7.0 / 21.7 \pm 2$ *

$\S / 32.36 \pm 3.95$

$44.8 \pm 8.8 / 40.2 \pm 4.7$ *

\begin{tabular}{c}
\hline AHI PCos/control \\
\hline Not specified \\
$22.5 \pm 6.0 / 6.7 \pm 1.4^{*}$ \\
$1.2 \pm 0.6 / 0.9 \pm 0.4$ \\
$\# \quad 2.1-126.7$
\end{tabular}

Not specified

$8.4 \pm 16.1$

$0.79 \pm 0.21 / 0.29 \pm 0.09$ *

$1.03 \pm 1.30 / 1.06 \pm 0.79$

$0.73 \pm 0.82 / 0.47 \pm 0.5$

$0.77 \pm 0.88 / 1.09 \pm 1.05$

$\S / 0.45 \pm 0.5$

$6.1 \pm 6.9 / 2.2 \pm 3.2$ *

Number of subjects, PCOS/control

Number of subjects

$53 / 452$
with OSA, PCOS/control

$18 / 18$

$8 / 1$

$42 / 17$

$52 / 21$

23

$0 / 0$

29/4*

16

50

33

38

12

$18 / 10$

$0 / 0$

22/11

$0 / 0$

$14 / 19$

$0 / 0$

$15 / 16$

$0 / 0$

$\S / 19$

$0 / 0$

$28 / 28$

$16 / 4$ *

${ }^{*} P<0.05$; "OSA(+)PCOS: $19.4 \pm 2.0$, OSA(-)PCOS: $2.0 \pm 0.4$, OSA(-)control: $2.3 \pm 0.3$; \#\#SB(+)PCOS: $29.8 \pm 3.4$, SDB(-)PCOS: $24.36 \pm 2.29$; 5 PCOS subjects: Group A: 14 IR(-)PCOS, age 15.24 \pm 0.93 , BMI 29.96 \pm 6.90 , AHI 0.68 \pm 0.69 ; Group B: 17 IR(+)PCOS, age $14.84 \pm 0.98, B M I$ l $36.58 \pm 5.67, A H I 0.76 \pm 0.47$. $\mathrm{AHI}$, apne-hypopnea index; BMI, body mass index; NP, nocturnal polysomnography; OSA, obstructive sleep apnea; PCOS, polycystic ovary syndrome.

for sleep apnea on Berlin Questionnaire, 63\% had poor sleep quality as assessed by the Pittsbugh Sleep Quality questionnaire and $45 \%$ had chronic daytime sleepiness as defined by the Epworth Sleepiness Scale (22). Women with PCOS reported more daytime sleepiness (as defined by Epworth Sleepiness Scale) than did controls in two other studies $(10,11)$. In one of these studies, the relation between PCOS and daytime sleepiness was reported to remain significant even after controlling for BMI (11). In a more recent study, it was found that a significant number of reproductive-age women with PCOS were at high risk for OSA based on Berlin Questionnaire (23). This increased risk was present in only obese women with PCOS, indicating that BMI was the only independent predictor of OSA risk on Berlin Questionnaire (23). Another study, investigating sleep disturbances using a modified version of Jenkins Sleep Questionnaire in a community-based sample of women with and without PCOS, showed that sleep disturbances were almost twice as common in women with PCOS compared with women of similar age without PCOS and difficulty achieving and maintaining sleep were most problematic (24). Findings about sleep architecture in adults with PCOS are conflicting. Significant differences in sleep-onset latency, sleep efficiency and percentage of REM were observed between women with PCOS and healthy controls in some but not all studies $(10,11,22)$. The discrepancy in the results of these studies may be because of the differences in the number and the BMI of the PCOS patients and the controls.

The pathophysiological mechanisms leading to increased risk of OSA in PCOS have not yet been identified. These mechanisms may relate to the main features of PCOS, including obesity, insulin resistance and hyperandrogenemia. Obesity is a well-established risk factor for OSA (25). Obesity may contribute to OSA through increased parapharyngeal fat deposition resulting upper airway narrowing, decreased thoracic compliance and reduced functional residual capacity due to the fat deposition in abdomen and around ribcage, and alterations in neural compensatory mechanisms and respiratory control system (9). PCOS is frequently associated with obesity (26), but some studies suggest that high prevalence of OSA in PCOS is not simply mediated through increased BMI. Vgontzas and coworkers showed that premenopausal women with PCOS were 30 times more likely to suffer from sleep disordered breathing than general population controls, and this difference remained significant even when corrected for BMI (11). Gopal and coworkers also found an increased prevalence of OSA in patients with http://www.endocrineconnections.org DOI: 10.1530/EC-17-0129 (c) 2017 The authors Published by Bioscientifica Ltd
This work is licensed under a Creative Commons Attribution-NonCommercial 4.0 International License. 


\begin{tabular}{l|l}
\hline Adult \\
Tock L, 2014 \\
ChatterjeebB, 2014 \\
Yang HP, 2009 \\
Tasali E, 2008 \\
Vgontzas AN, 2006 \\
Gopal M, 2002 \\
Fogel RB, 2001 \\
Vgontzas AN, 2001 \\
RE Model for Subgroup \\
Adolescent
\end{tabular}

Figure 2

Forest plot of OSA prevalence in PCOS patients.
PCOS, but there was no correlation between severity of OSA and the degree of obesity (12). In patients with PCOS, obesity is typically central in nature (increased waist-tohip ratio) and central obesity has been shown to confer a higher risk for OSA than generalized obesity (27). This was demonstrated in a study by Fogel and coworkers (10). In this study, waist-to-hip ratio was significantly correlated with AHI. Consistent with these findings, Chatterjee and coworkers showed that mean waist circumference, also a marker for central obesity, was not only higher in PCOS patients with OSA than the ones without OSA, but also correlated positively with the severity of OSA (13). All these studies have included mostly obese women, but in a study by Yang and coworkers, including only nonobese women, even though the AHI in the PCOS patients was not high enough to meet the OSA criteria, women with PCOS had more hypopneic-apneic events during sleep than the age- and BMI-matched control group, showing that risk of OSA in patients with PCOS is still increased without the direct effect of obesity (28).

There are several reports suggesting that androgen levels can directly influence the prevalence and severity of OSA in both men and women $(29,30,31)$. High androgen levels may predispose to the development of OSA by increasing soft tissue deposition in the pharynx and effects on the ventilatory control mechanism, thereby influencing the patency of the pharynx and making it more collapsible during sleep (32). Androgen excess is a defining feature of PCOS, but its role in the pathogenesis of OSA in women with PCOS remains controversial. In some studies, there were no significant correlations between androgen levels and the severity of OSA $(11,22,33)$. In contrast, Fogel and coworkers showed that AHI of obese women with PCOS correlated with both total and free testosterone levels (10). Yang and coworkers demonstrated that even in lean women with PCOS without diagnostic criteria for OSA, total testosterone levels were positively correlated with AHI (28). An association between the occurence of hyperandrogenemia and the presence of OSA was also found in a study by Tock and coworkers (14). In this study, including 38 premenopausal women with PCOS, $50 \%$ of patients with high free testosterone levels were also affected by OSA, compared with only $15 \%$ of those without hyperandrogenemia. The AHI showed positive and significant correlation with free testosterone levels, even after adjusting for obesity. Chatterjee and coworkers also found a positive correlation between the severity of sleep disordered breathing and free testosterone levels (13).

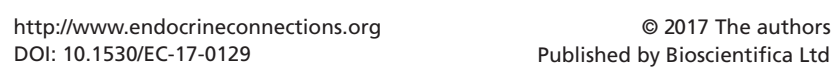


OR $[95 \% \mathrm{Cl}]$

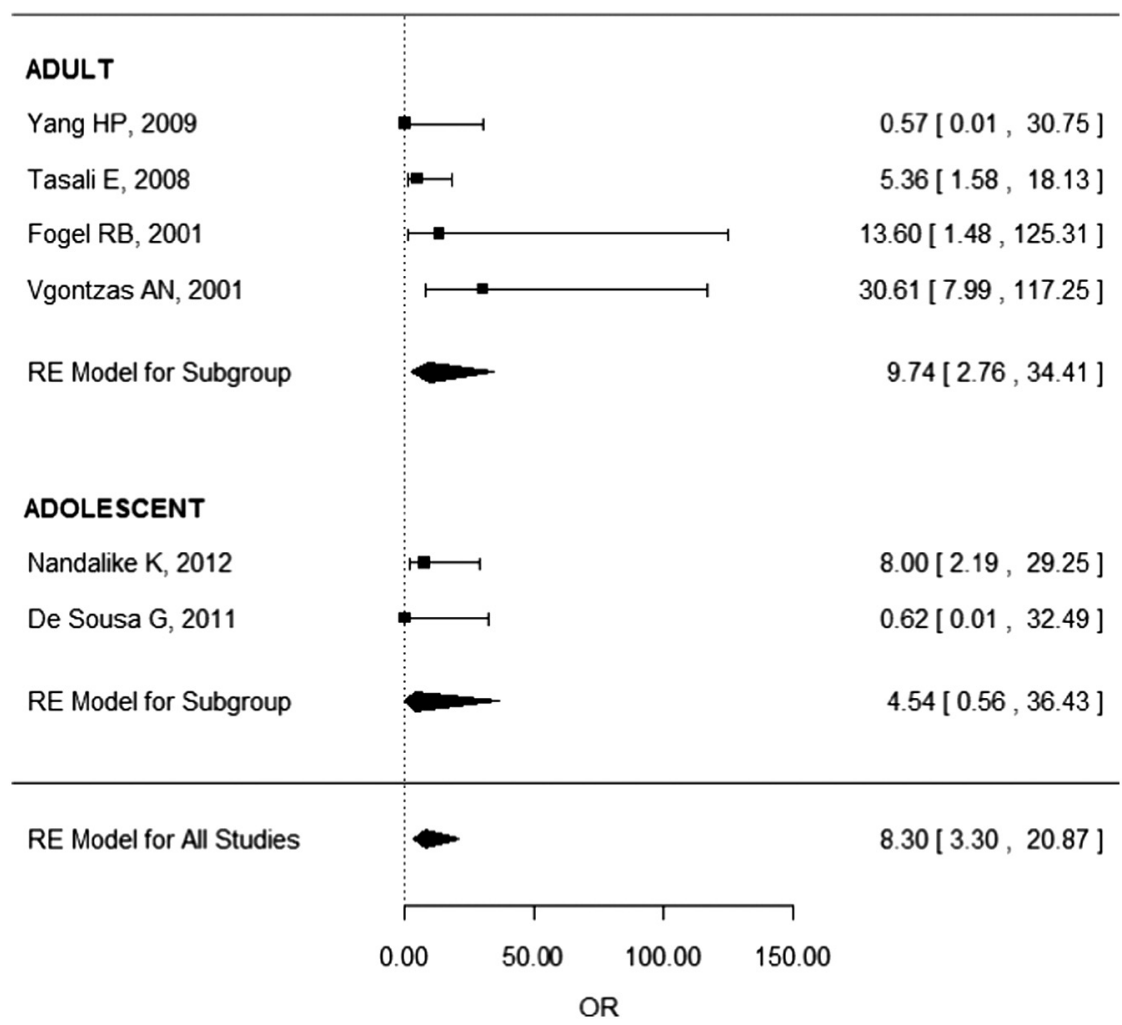

Figure 3

Risk of OSA in PCOS patients.
Taken together, these data may suggest that androgens can influence the development of OSA in patients with PCOS.

Beside the higher levels of androgens, women with PCOS also have lower levels of progesterone. Data derived from the studies in which sleep was evaluated in relation to pregnancy, phase of menstrual cycle, menopause or hormone replacement therapy, suggests that progesterone has a protective effect against OSA $(34,35,36)$. Progesterone is considered to have a stimulatory effect on respiratory drive and also enhance the activity of upper airway dilator muscle $(37,38)$. Low concentrations of progesterone due to chronic anovulation in PCOS, may contribute to the high prevalence of OSA in this disorder, but progesterone levels among women with PCOS and OSA have not been systematically evaluated in the studies.

Insulin resistance is considered to be another defining feature of PCOS (39). There is also strong evidence that the presence and the severity of OSA per se is associated with impaired glucose tolerance and insulin resistance, independent of BMI or other measures of obesity $(8,40$, 41). Intermittent hypoxia, elevated activity of sympathetic nervous system and hypothalamo-pituitary axis and release of proinflammatory cytokines have all been proposed as potential mechanisms involved in the association of OSA and insulin resistance and glucose intolerance (42). Studies in adults have suggested a relationship between OSA and parameters of glucose metabolism in women with PCOS. Vgontzas and coworkers found that PCOS patients had significantly higher plasma insulin levels and a lower glucose-to-insulin ratio than those who did not have clinically significant OSA, independently of age, BMI and testosterone levels $(11,43)$. Accordingly, Chatterjee and coworkers found that fasting blood sugar was significantly higher in patients having PCOS with SDB (13). Consistent with these findings, Tasali and coworkers demonstrated a correlation between the risk and severity of OSA and insulin levels and measures of glucose tolerance $(22,33)$. They showed that fasting insulin concentration and HOMA index, measures of insulin resistance, were markedly higher in PCOS women with OSA than in PCOS women without OSA, even after controlling for age, BMI and ethnicity and argued that the insulin resistance in PCOS was determined by the presence of OSA (33). In an other study in young, obese women with PCOS, successful treatment of OSA with continous positive airway pressure modestly improved insulin sensitivity after controlling for BMI (44). Above findings suggest that although it is difficult to establish the exact direction, there may be an association between the insulin resistance in PCOS and the occurence and the severity of OSA. Eventually, it is http://www.endocrineconnections.org DOI: 10.1530/EC-17-0129
() 2017 The authors Published by Bioscientifica Ltd
This work is licensed under a Creative Commons Attribution-NonCommercial 4.0 International License. 
plausible that in the presence of significant OSA, insulin resistance will be more severe in patients with PCOS.

There are several limitations of this study, which should be addressed. Most of the included studies are cross-sectional in design and have a small sample size. Secondly, one study in adolescents was based on patients referred for polysomnography (15). Nevertheless, we chose not to exclude the study because the total number of the studies relating to PCOS and OSA was very limited. Thirdly, the diagnostic criteria for OSA was not uniform among studies. In one included study, AHI more than 10 events per hour was considered as diagnostic for OSA (11). In other included studies, diagnostic AHI levels were more than 5 events per hour. There were also differences in the definitions of apnea and hypopnea among the included studies. Fourthly, there were also differences in the diagnostic criterias of PCOS among studies. One study did not specify the criteria used to diagnose PCOS (12). In other included studies, the diagnosis of PCOS was based on either Rotterdam or NIH criteria. Finally, because of the very small number of the studies with available descriptive statistics, we were not able to perform metaregression analysis to determine the effects of the measures of obesity, insulin resistance and hyperandrogenemia on the association between PCOS and OSA.

To confirm the findings of this study, further studies focusing on community-based cohorts and free from clinical referral bias, would be useful to determine whether PCOS is an independent risk factor for OSA. Longitudinal studies, ideally with a large number of cases and controls, are needed to determine when OSA develops and which factors are involved in the pathogenesis of OSA in patients with PCOS. However, considering the fact that polysomnography is a complex, costly and time-consuming investigation requiring the overnight hospitalization of the patient, such studies are extremely difficult to perform.

In conclusion, this meta-analysis suggests that risk of OSA is increased in adult women with PCOS. The results of available studies are conflicting, but it seems that central obesity, hyperandrogenemia and insulin resistance, either alone or in concert, could be implicated in the development of OSA in women with PCOS. Considering the fact that successful treatment of OSA improves cardiometabolic function, it is of importance at this moment to diagnose OSA in patients with PCOS who already face a lifelong risk of cardiovascular disease and type 2 diabetes. Therefore, it would be wise for physicians who diagnose and treat PCOS to screen patients with clinical questionnaires for clinical features and symptoms associated with OSA and refer the ones who deemed to be at risk for further evaluation with overnight polysomnography.

\section{Declaration of interest}

The authors declare that there is no conflict of interest that could be perceived as prejudicing the impartiality of the research reported.

\section{Funding}

This work did not receive any specific grant from any funding agency in the public, commercial, or not-for-profit sector.

\section{References}

1 Azziz R, Woods KS, Reyna R, Key TJ, Knochenhauer ES \& Yildiz BO. The prevalence and features of the polycystic ovary syndrome in an unselected population. Journal of Clinical Endocrinology and Metabolism 200489 2745-2749. (doi:10.1210/jc.2003-032046)

2 Yildiz BO, Bozdag G, Yapici Z, Esinler I \& Yarali H. Prevalence, phenotype and cardiometabolic risk of polycystic ovary syndrome under different diagnostic criteria. Human Reproduction 201227 3067-3073. (doi:10.1093/humrep/des232)

3 Legro RS, Arslanian SA, Ehrmann DA, Hoeger KM, Murad MH, Pasquali R \& Welt CK. Diagnosis and treatment of polycystic ovary syndrome: an Endocrine Society clinical practice guideline. Journal of Clinical Endocrinology and Metabolism 201398 4565-4592. (doi:10.1210/jc.2013-2350)

4 Fauser BC, Tarlatzis BC, Rebar RW, Legro RS, Balen AH, Lobo R, Carmina E, Chang J, Yildiz BO, Laven JS, et al. Consensus on women's health aspects of polycystic ovary syndrome (PCOS): the Amsterdam ESHRE/ASRM-Sponsored 3rd PCOS Consensus Workshop Group. Fertility and Sterility 201297 28.e25-38.e25. (doi:10.1016/j. fertnstert.2011.09.024)

5 Caples SM, Gami AS \& Somers VK. Obstructive sleep apnea. Annals of Internal Medicine 2005142 187-197. (doi:10.7326/0003-4819-142-3200502010-00010)

6 Peppard PE, Young T, Palta M \& Skatrud J. Prospective study of the association between sleep-disordered breathing and hypertension. New England Journal of Medicine 2000342 1378-1384. (doi:10.1056/ NEJM200005113421901)

7 Peker Y, Carlson J \& Hedner J. Increased incidence of coronary artery disease in sleep apnoea: a long-term follow-up. European Respiratory Journal 200628 596-602. (doi:10.1183/09031936.06.00107805)

8 Punjabi NM \& Polotsky VY. Disorders of glucose metabolism in sleep apnea. Journal of Applied Physiology 200599 1998-2007. (doi:10.1152/ japplphysiol.00695.2005)

9 Punjabi NM. The epidemiology of adult obstructive sleep apnea. Proceedings of the American Thoracic Society 20085

136-143. (doi:10.1513/pats.200709-155MG)

10 Fogel RB, Malhotra A, Pillar G, Pittman SD, Dunaif A \& White DP. Increased prevalence of obstructive sleep apnea syndrome in obese women with polycystic ovary syndrome. Journal of Clinical Endocrinology and Metabolism 200186 1175-1180. (doi:10.1210/jcem.86.3.7316)

11 Vgontzas AN, Legro RS, Bixler EO, Grayev A, Kales A \& Chrousos GP. Polycystic ovary syndrome is associated with obstructive sleep apnea and daytime sleepiness: role of insulin resistance. Journal of Clinical Endocrinology and Metabolism 200186 517-520. (doi:10.1210/jcem.86.2.7185)

12 Gopal M, Duntley S, Uhles M \& Attarian H. The role of obesity in the increased prevalence of obstructive sleep apnea syndrome in patients with polycystic ovarian syndrome. Sleep Medicine 20023 401-404. (doi:10.1016/S1389-9457(02)00033-3)

13 Chatterjee B, Suri J, Suri JC, Mittal P \& Adhikari T. Impact of sleepdisordered breathing on metabolic dysfunctions in patients with polycystic ovary syndrome. Sleep Medicine 201415 1547-1553. (doi:10.1016/j.sleep.2014.06.023)

14 Tock L, Carneiro G, Togeiro SM, Hachul H, Pereira AZ, Tufik S \& Zanella MT. Obstructive sleep apnea predisposes to nonalcoholic Fatty

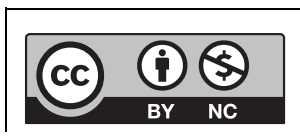

This work is licensed under a Creative Commons Attribution-NonCommercial 4.0 International License. 
liver disease in patients with polycystic ovary syndrome. Endocrine Practice 201420 244-251. (doi:10.4158/EP12366.OR)

15 Nandalike K, Agarwal C, Strauss T, Coupey SM, Isasi CR, Sin S \& Arens R. Sleep and cardiometabolic function in obese adolescent girls with polycystic ovary syndrome. Sleep Medicine 201213 1307-1312. (doi:10.1016/j.sleep.2012.07.002)

16 Higgins JPT \& Green S. Cochrane Handbook for Systematic Reviews of Interventions Version 5.1.0. London, UK: The Cochrane Collaboration, 2011. (available at: http://training.cochrane.org/handbook)

17 de Sousa G, Schlüter B, Menke T, Trowitzsch E, Andler W \& Reinehr T. A comparison of polysomnographic variables between obese adolescents with polycystic ovarian syndrome and healthy, normal-weight and obese adolescents. Sleep and Breathing 201014 33-38. (doi:10.1007/s11325-009-0276-0)

18 de Sousa G, Schlüter B, Menke T, Trowitzsch E, Andler W \& Reinehr T. Longitudinal analyses of polysomnographic variables, serum androgens, and parameters of glucose metabolism in obese adolescents with polycystic ovarian syndrome. Sleep and Breathing 201216 1139-1146. (doi:10.1007/s11325-011-0620-z)

19 de Sousa G, Schlüter B, Buschatz D, Menke T, Trowitzsch E, Andler W $\&$ Reinehr T. The impact of insulin resistance and hyperandrogenemia on polysomnographic variables in obese adolescents with polycystic ovarian syndrome. Sleep and Breathing 201216 169-175. (doi:10.1007/ s11325-010-0469-6)

20 de Sousa G, Schlüter B, Menke T, Trowitzsch E, Andler W \& Reinehr T. A comparison of polysomnographic variables between adolescents with polycystic ovarian syndrome with and without the metabolic syndrome. Metabolic Syndrome and Related Disorders 20119 191-196. (doi:10.1089/met.2010.0081)

21 Nandalike K, Strauss T, Agarwal C, Coupey SM, Sin S, Rajpathak S, Cohen HW \& Arens R. Screening for sleep-disordered breathing and excessive daytime sleepiness in adolescent girls with polycystic ovarian syndrome. Journal of Pediatrics 2011159 591-596. (doi:10.1016/j.jpeds.2011.04.027)

22 Tasali E, Van Cauter E \& Ehrmann DA. Relationships between sleep disordered breathing and glucose metabolism in polycystic ovary syndrome. Journal of Clinical Endocrinology and Metabolism 200691 36-42. (doi:10.1210/jc.2005-1084)

23 Mokhlesi B, Scoccia B, Mazzone T \& Sam S. Risk of obstructive sleep apnea in obese and nonobese women with polycystic ovary syndrome and healthy reproductively normal women. Fertility and Sterility 2012 97 786-791. (doi:10.1016/j.fertnstert.2011.12.024)

24 Moran LJ, March WA, Whitrow MJ, Giles LC, Davies MJ \& Moore VM. Sleep disturbances in a community-based sample of women with polycystic ovary syndrome. Human Reproduction 201530 466-472. (doi:10.1093/humrep/deu318)

25 Young T, Palta M, Dempsey J, Skatrud J, Weber S \& Badr S. The occurrence of sleep-disordered breathing among middle-aged adults. New England Journal of Medicine 1993328 1230-1235. (doi:10.1056/ NEJM199304293281704)

26 Salehi M, Bravo-Vera R, Sheikh A, Gouller A \& Poretsky L. Pathogenesis of polycystic ovary syndrome: what is the role of obesity? Metabolism 200453 358-376. (doi:10.1016/j.metabol.2003.10.005)

27 Simpson L, Mukherjee S, Cooper MN, Ward KL, Lee JD, Fedson AC, Potter J, Hillman DR, Eastwood P, Palmer LJ, et al. Sex differences in the association of regional fat distribution with the severity of obstructive sleep apnea. Sleep 201033 467-474. (doi:10.1093/ sleep/33.4.467)

28 Yang H-P, Kang J-H, Su H-Y, Tzeng C-R, Liu W-M \& Huang S-Y. Apnea-hypopnea index in nonobese women with polycystic ovary syndrome. International Journal of Gynaecology and Obstetrics 2009105 226-229. (doi:10.1016/j.ijgo.2009.02.004)

29 Dexter DD \& Dovre EJ. Obstructive sleep apnea due to endogenous testosterone production in a woman. Mayo Clinic Proceedings 199873 246-248. (doi:10.1016/S0025-6196(11)64467-4)

30 Cistulli PA, Grunstein RR \& Sullivan CE. Effect of testosterone administration on upper airway collapsibility during sleep. American Journal of Respiratory and Critical Care Medicine 1994149 530-532. (doi:10.1164/ajrccm.149.2.8306057)

31 Zhou XS, Rowley JA, Demirovic F, Diamond MP \& Badr MS. Effect of testosterone on the apneic threshold in women during NREM sleep. Journal of Applied Physiology 200394 101-107. (doi:10.1152/ japplphysiol.00264.2002)

32 Eckert DJ \& Malhotra A. Pathophysiology of adult obstructive sleep apnea. Proceedings of the American Thoracic Society 20085 144-153. (doi:10.1513/pats.200707-114MG)

33 Tasali E, Van Cauter E, Hoffman L \& Ehrmann DA. Impact of obstructive sleep apnea on insulin resistance and glucose tolerance in women with polycystic ovary syndrome. Journal of Clinical Endocrinology 200893 3878-3884. (doi:10.1210/jc.2008-0925)

34 Brownell LG, West P \& Kryger MH. Breathing during sleep in normal pregnant women. American Review of Respiratory Disease 1986133 38-41. (doi:10.1164/arrd.1986.133.1.38)

35 Driver HS, McLean H, Kumar DV, Farr N, Day AG \& Fitzpatrick MF The influence of the menstrual cycle on upper airway resistance and breathing during sleep. Sleep 200528 449-456. (doi:10.1093/ sleep/28.4.449)

36 Shahar E, Redline S, Young T, Boland LL, Baldwin CM, Nieto FJ, O'Connor GT, Rapoport DM \& Robbins JA. Hormone replacement therapy and sleep-disordered breathing. American Journal of Respiratory and Critical Care Medicine 2003167 1186-1192. (doi:10.1164/ rccm.200210-1238OC)

37 Pien GW \& Schwab RJ. Sleep disorders during pregnancy. Sleep 2004 27 1405-1417. (doi:10.1093/sleep/27.7.1405)

38 Popovic RM \& White DP. Upper airway muscle activity in normal women: influence of hormonal status. Journal of Applied Physiology 199884 1055-1062.

39 Dunaif A, Segal KR, Futterweit W \& Dobrjansky A. Profound peripheral insulin resistance, independent of obesity, in polycystic ovary syndrome. Diabetes 198938 1165-1174. (doi:10.2337/ diab.38.9.1165)

40 Punjabi NM, Shahar E, Redline S, Gottlieb DJ, Givelber R \& Resnick HE. Sleep-disordered breathing, glucose intolerance, and insulin resistance: the sleep heart health study. American Journal of Epidemiology 2004160 521-530. (doi:10.1093/aje/kwh261)

41 Lévy P, Bonsignore MR \& Eckel J. Sleep, sleep-disordered breathing and metabolic consequences. European Respiratory Journal 200934 243-260. (doi:10.1183/09031936.00166808)

42 Van Cauter E. Sleep disturbances and insulin resistance. Diabetes Medical 201128 1455-1462. (doi:10.1111/j.1464-5491.2011.03459.x)

43 Vgontzas AN, Trakada G, Bixler EO, Lin HM, Pejovic S, Zoumakis E, Chrousos GP \& Legro RS. Plasma interleukin 6 levels are elevated in polycystic ovary syndrome independently of obesity or sleep apnea. Metabolism 200655 1076-1082. (doi:10.1016/ j.metabol.2006.04.002)

44 Tasali E, Chapotot F, Leproult R, Whitmore H \& Ehrmann DA. Treatment of obstructive sleep apnea improves cardiometabolic function in young obese women with polycystic ovary syndrome. Journal of Clinical Endocrinology and Metabolism 201196 365-374. (doi:10.1210/jc.2010-1187)

Received in final form 28 June 2017

Accepted 24 July 2017

Accepted Preprint published online 24 July 2017 http://www.endocrineconnections.org

DOI: 10.1530/EC-17-0129

2017 The authors Published by Bioscientifica Ltd

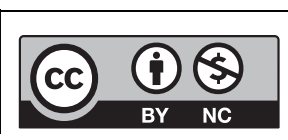

This work is licensed under a Creative Commons Attribution-NonCommercial 4.0 International License. 